\title{
Blasenkrebs
}

\section{Rauchen verkürzt rezidivfreie Zeit}

- Rauchen lässt nach der Therapie eines Blasenkarzinoms den Tumor schneller wieder wachsen. Diesen Zusammenhang hat ein Forscherteam aus den Niederlanden nachgewiesen [Lammers R JM et al. J Eur Urol 2011; 60: 713-20]. Die Wissenschaftler analysierten Daten von 718 Patienten, die im Rahmen einer prospektiven randomisierten Phase-III-Studie nach Diagnose und Therapie eines nicht muskelinvasiven Harnblasenkarzinoms im Durchschnitt zweieinhalb Jahre lang begleitet worden waren. Dazu teilten sie die Patienten in Raucher, ehemalige Raucher und Nichtraucher ein.

284 Patienten erlitten ein Rezidiv, durchschnittlich nach 19,6 Monaten. Das Rauchen stellte sich dabei als eindeutiger Risikofaktor heraus: Es förderte das erneute Tumorwachstum - nach 2,5 Jahren hatten 53,2 \% der Raucher, aber nur 37,7\% der Nichtraucher ein Rezidiv - und verkürzte deutlich die Zeit bis zum Wiederauftreten. Vor allem bei Patienten mit mehr als 35 Packungsjahren schlug sich dieser Zusammenhang nieder $(p=0,005)$. Faktoren wie vorherige Rezidive $(p<0,003)$ und mehrere Primärtumore in der Blase $(p<0,004)$ verkürzten die rezidivfreie Zeit ebenfalls.

Die Autoren vermuten, dass der Rauch-Status die Effektivität der Chemotherapie beeinflusst, denn das Chemotherapieregime war bei allen Krebspatienten identisch. Das Ergebnis der Studie unterstreiche nochmals, so die Autoren, wie wichtig es ist, dass die Patienten mit dem Rauchen aufhören. Deshalb müsse der behandelnde Arzt seinen Tumorpatienten noch ausführlicher informieren und eindringlich vor den Risiken warnen.

Dr. Dagmar Kraus

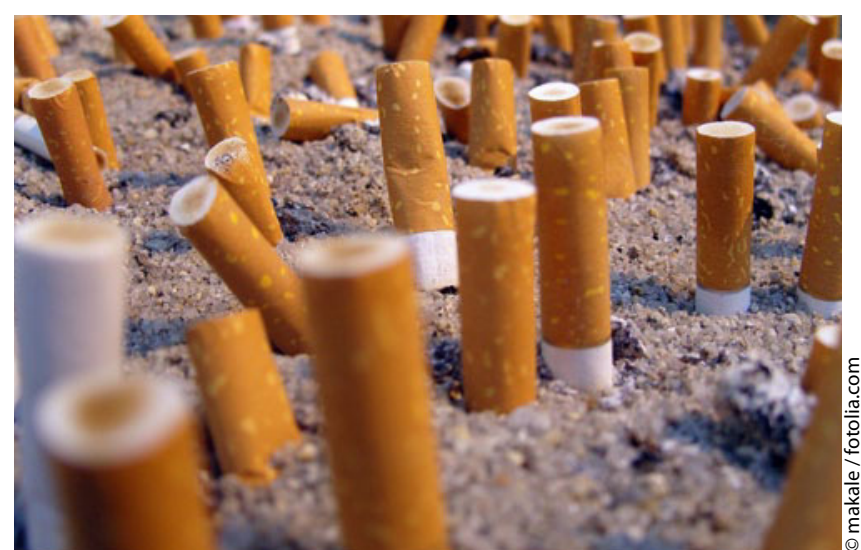

Rauchen begünstigt neben der Entstehung auch die Rezidivneigung von Blasenkrebs.

\section{ACHTUNG:}

\section{Hier muss der}

Dummy durch

eine Anzeige

ersetzt werden !! 